\title{
BMJ Open Can nurse teachers manage student incivility by guided democracy? A grounded theory study
}

\author{
Mostafa Rad, ${ }^{1}$ Hossein Karimi Moonaghi, ${ }^{2,3}$ Eshagh Ildarabadi ${ }^{4}$
}

To cite: Rad M, Karimi Moonaghi H, Ildarabadi E. Can nurse teachers manage student incivility by guided democracy? A grounded theory study. BMJ Open 2017;7:e014639. doi:10.1136/ bmjopen-2016-014639

- Prepublication history for this paper is available online. To view these files please visit the journal online (http://dx.doi. org/10.1136/bmjopen-2016014639)

Received 17 October 2016 Revised 19 May 2017

Accepted 22 May 2017

\section{(a) CrossMark}

${ }^{1}$ School of Nursing and Midwifery,Sabzevar University of Medical Sciences, Sabzevar, Iran ${ }^{2}$ Evidence- Based Caring Research Center, Department of Medical Surgical Nursing,

School of Nursing and

Midwifery, Mashhad University of Medical Sciences, Mashhad, Iran

${ }^{3}$ Department of Medical Education, School of Medicine, Mashhad University of Medical Sciences, Mashhad, Iran ${ }^{4}$ Department of Nursing, Esfarayen Faculty of Medical Sciences, Esfarayen, Iran

Correspondence to Dr Hossein Karimi Moonaghi; karimih2001@yahoo.com

\begin{abstract}
IntroductionManaging incivility in academic settings is among the basic concerns and challenges of most educational systems, including nursing education. Incivility management cannot be considered devoid of disruptive behaviors. However, incivility management is a complexphenomenon upon which few studies are conducted.
\end{abstract}

ObjectivesThe present study aims at discovering teachers and students' experiences regarding incivility and developing an approach to manage nursing students' incivility.

DesignThe present study was conducted based on the qualitative research design of the grounded theory methodology.

SettingsThis study was conducted at schools of nursing in academic settings in Iran.

ParticipantsStudy participants in the present study include nurse teachers $(\mathrm{N}=20)$ and nursing students $(\mathrm{N}=9)$.

Method In-depth semi-structured interviews were conducted using theoretical and purposive sampling. Constant comparative analysis was used for data analysis. Results The results include four main categories; (1) deterioration of learning; (2) dominant individual and organisational culture; (3) guided democracy; and (4) movement toward professionalism. Guided democracy is recognised as the main basic psychosocial process for incivility management.

Conclusions Incivility management is pursued to help learners develop professional performance. As indicated by the results of the present study, guided democracy is an effective strategy for incivility management in nursing education.

\section{INTRODUCTION}

Interacting with students, nurse teachers usually encounter numerous challenging behaviours including incivility as one major category. Academic incivility may include any words or actions with negative impacts on the health of students and teachers, leading to impoverished faculty-student interaction and a complicated learning-teaching process. ${ }^{1}$ Incivility behaviours are mainly characterised by rudeness and inconsiderate treatment of others, disrespecting others, imposing beliefs on others as well as behaviours with negative effects on initiator-recipient interaction. ${ }^{2}$
Strengths and limitations of the present study

- This study describes a carefully developed and open but structured approach in researching incivility management in nursing education.

- Grounded theory method can provide in-depth identification, description and explanation of interactional processes between nurse teachers and students.

- Collecting data from a wide variety of teachers and students provided new insights into incivility management.

- Incivility management is a complex phenomenon whose comprehensive investigation requires further study.

- In this study it was not possible to determine the off-campus sources of uncivil behaviour.

Students' incivility is becoming a progressive challenge for nursing academia. ${ }^{3}$ These behaviours are being debated in global nursing education, and the National Organisation of Nursing has declared it as a problem for nurse teachers. Swinney and colleagues contend that schools as part of the whole society are now far from being immune to incivility. Additionally, no one may deny the fact that such behaviours are observable in university students too. ${ }^{4}$ Such behaviours are likely to affect teachers with discouragement, stress, shock and concern unless they are recognised and managed in time. ${ }^{56}$ The consequent outcomes may appear both shortterm and long-term. Teachers' physical and psychological reactions, diffidence and lack of satisfaction towards their performance regarding teaching content and style and the negative consequence imposed on teaching processes are obvious examples of students' incivility. $^{78}$ Students' incivility can negatively affect managers' performance leading to stress and performance disruption. ${ }^{9} 10$ Another consequence is the interference of incivility with classroom discipline, particularly during team-based learning activities in small groups where teachers expect active 
and effective participation from learners. ${ }^{11}$ However, being indifferent to students' incivilty in the educational context results in future negative consequences including students' unjustified job quitting and bullying colleagues. ${ }^{12}$ The consequences of uncivil behaviours are widespread and offen long-term, including physiological symptoms, anxiety, lowered self-esteem and use of alcohol or drugs. In addition, the victims of incivility suffer from cognitive defects, personality changes, weak communication skills, deterioration of self-confidence, stress, violence and workplace complications. ${ }^{13}$

Schools of nursing undoubtedly play a major role in developing and guaranteeing the professional qualifications of their graduates. ${ }^{14}$ Also, nurse teachers have a unique position in helping students to develop safer and more well-advised ethical behaviours. ${ }^{15}$ Adherence to the principles and ethical standards of the nursing profession guide teachers to train students with high qualifications. Educators need to upgrade their competency to manage students' uncivil behaviours. ${ }^{16}$ The quality of management needs a backbone of models and theories because the achievement and development of a professional identity towards the social interactions between educators and students cannot emerge unsystematically. ${ }^{17}$ The high prevalence of incivility in nursing and other clinical professions ${ }^{18}$ requires further studies in the field of uncivil behaviours. ${ }^{19}$

Educators are normally expected to tackle behaviours affecting their teaching through their experiences and to develop effective strategies to address them. Researchers often put forward solutions for managing incivility, including problem-solving strategies, ${ }^{20}$ violence prevention models ${ }^{21}$ and counselling plus faculty-staff rapport. ${ }^{9}$ As the context of some studies conducted so far has been in Western countries, the researchers decided to investigate the issue in the Middle East and to develop deeper explanations for incivility management in these contexts.

The present study was conducted to discover teachers' and students' experiences regarding incivility among students and to develop an approach to managing nursing students' incivility.

\section{METHODS \\ Design}

Grounded theory methodology was used to achieve the objectives of this study.

\section{Data collection}

We collected and analysed data from September 2014 to December 2016. The major interview questions were initially expressed as:

\footnotetext{
"What is your conception of students' incivility?"

"Could you please describe a day in your teaching when you faced students' incivility?" and

"Please describe how you managed students' incivility".
}

The interviewer probed participant responses by using questions or statements such as "Could you say something more about that?" or "What did you think then?" Interviews then became more specific with questions based on participants' answers in order to complete the emerged concepts and categories. Face-to-face in-depth interviews were conducted at students' or researchers' offices; each interview took 60-90 min. Interviews were recorded by a digital recorder and transcribed verbatim. The number of participants was 29 (20 nurse teachers and 9 nursing students) and five further interviews were planned for further reference and enrichment of the data if needed for a total of 34 interviews (table 1). Post-interview comment sheets (PICS) contributed considerably to data analysis and formation of tentative categories before data saturation and establishment of the results. Interviews were conducted by MR under the supervision of HKM.

\section{Setting}

The study was conducted on both teachers and students of Schools of Nursing in five universities in Iran.

\section{Sample}

The participants were 20 nurse teachers and nine nursing students. To be included as a participant, teachers needed a minimum of 1 year experience in training nurses and students had to pass their first year of education in nursing. Maximum variety was sought for participants (Table 1) until the saturation point was reached in data collection. Participants were initially selected through purposive sampling, and theoretical sampling was then used on analysis.

\section{Analysis of data}

Consistent with the grounded theory method, interviews were analysed one by one. Data were analysed according to the Strauss and Corbin method ${ }^{22}$ and MAXQDA was used for data management. Therefore, data coding was carried through three stages of open, axial and selective coding processes. During open coding, each transcript was read many times and codes were generated from participants' words and researchers' constructs. For example, the code 'teacher's low self-confidence' was generated by the researcher based on participant's comment that "... I wasn't really able to establish rapport with that particular student so that he/she accepts me officially as his/ her teacher; I felt that I wasn't really accepted there ...".

Codes conceptually similar in nature or related in meaning were grouped in categories. The codes and categories from each interview were compared with those from other interviews in order to identify common links. Categories were related to their subcategories in axial coding. Coding was done around the axis of a category (ie, linking categories at the level of properties and dimensions). In this stage, the relationships among concepts and the link among action-interaction within a framework of concepts are identified. These concepts explain what interactions are occurring, and why and 
Table 1 Participants' details

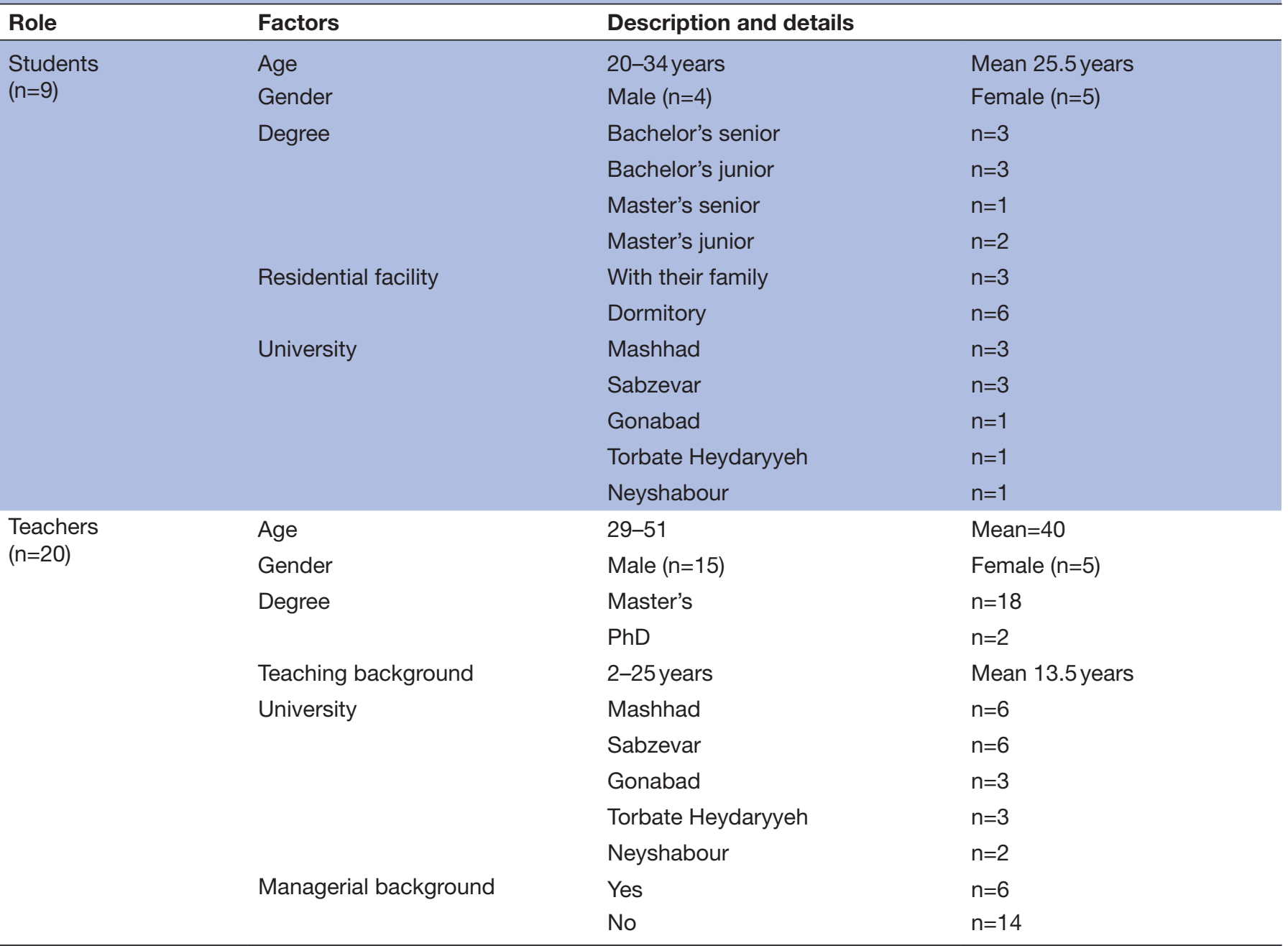

what consequences are happening because of actioninteraction. For instance, teachers' and students' poor practice was identified. The process of integrating and refining the main categories occurred in selective coding (table 2).

Table 2 Categories and subcategories of incivility management in nursing education

\begin{tabular}{ll}
\hline Main categories & Subcategories \\
\hline Deterioration of learning & 1. Students' immaturity \\
Dominant individual and organisational culture & 2. Teachers' and students' poor practice \\
1. Students' personal characteristics and beliefs & 2. Teachers' personal characteristics and beliefs \\
Guided democracy & $\begin{array}{l}\text { 3. University nature } \\
\text { 4. Variation of norms for students and teachers }\end{array}$ \\
& 1. Teachers' accountability \\
2. Cordiality strategies \\
3. Teaching-learning strategies \\
4. Exigency decision-making \\
5. Establishment of a collaborative atmosphere in education \\
6. Intelligent self-regulation
\end{tabular}

1. Students' immaturity

2. Teachers' and students' poor practice

1. Students' personal characteristics and beliefs

2. Teachers' personal characteristics and beliefs

4. Variation of norms for students and teachers

3. Teaching-learning strategies

4. Exigency decision-making

6. Intelligent self-regulation

1. Students' educational engagement

3. Promotion of teacher-student interaction

4. The win-win consequence for both parties 
When analysing the data, the researcher asked himself a few questions to do the analysis more correctly-for example, what was the main concern of participants and how did they treat this concern to solve it?

\section{Ethical considerations}

Permission to conduct this study was issued by the Ethics Committee of Mashhad University of Medical Sciences based on a formal letter of introduction from the Vice Dean for Research of University of Medical Sciences, serving as the legal authority in this area (No. 921903, 2014/08/03). We have placed emphasis on participants' confidentiality, their informed consent, right to exit from the study at any time, the selection of time and place of interview and anonymity. Permission, as written informed consent, was sought from the participants for the audiotaped interviews.

\section{RESULTS}

The categories that emerged from data analysis are all related to nursing teachers' and students' experiences about incivility. After the reduction and integration of similar codes, four main categories and 16 subcategories were found. All categories were related to each other and revealed the pattern of incivility management. In fact, these categories describe the phenomenon of incivility and the variables affecting it. Therefore, in response to the research question, we can say that teachers use guided democracy as a main strategy for incivility management in nursing education due to: (1) deterioration of learning; (2) dominant individul and organisational culture; and (3) movement to professionalism. These concepts help the reader to understand the reality of incivility in nursing education (see table 2). This paper presents data about incivility in nursing education, including narratives from participants.

\section{Deterioration of learning}

The deterioration of learning was the main concern of participants as the causal condition for the guided democracy. Defective academic performance caused deterioration of learning, which consisted of two subcategories: (1) students' immaturity, (2) teachers' and students' poor practice.

\section{Students' immaturity}

Students' intellectual maturity was conceived to play an important role in managing incivility through resolving the existing conflicts. Lack of intellectual maturity and unfamiliarity with the characteristics of an ideal student led to poor studentship. Misinformed by their seniors, knowledge deficit about university rules, desire for others' attention, misunderstanding and misinterpretation of teachers' dialogue, poor anticipation, low understanding of the situations and teachers' authority all contributed to students' immaturity. Teachers believed that both incivility management and occurrence were affected by students' unfamiliarity with the ethical position and expectations. In addition, low motivation, cynicism and priority of extracurricular activities reinforced incivility management. Note a participant's comments below:

“... when students enter the university as freshmen, they are still acting as high schoolers thinking that they can be free and relaxed here too; they treat people around them, their friends and professors the way they wish ..." (Teacher 3)

\section{Teachers' and students' poor practice}

Generally, teachers' poor practice and its consequential effects consisted of their low experience and inability to communicate with students. Being unskilled for classroom and clinical training, inappropriate use of media, trial-and-error management strategies, defective control over assignments, teaching with little preparation and through less effective methods were all among the stated reasons for unsuccessful incivility management. In some cases, lack of communication due to an inflated opinion of oneself and a sense of superiority were among the reasons behind disregarding students' disruptive behaviours. Note a participant's comments below:

“... I wasn't really able to establish rapport with that particular student so that he/she accepts me officially as his/her teacher; I felt that I wasn't really accepted there ..." (Teacher 5)

The concept of poor practice was characterised, in teachers' words, with poor teaching and learning, disturbance of the classroom order and endangered education in general. Some symptoms of defective learning were reported to be: disinclination towards gaining more knowledge and attitudes towards the field of nursing, passive learning of the skills, low preparation, delayed assignments and lowered commitment to scientific principles in practice. Examples of chaotic teaching were also reported to be: losing control over students, unskilled time management, scattered incivility, disordered classes, absenteeism, frequent late comers, frequent interruptions, no preparation for examinations and closing teaching sessions too early. Note a participant's comments below:

"... Some young teachers cannot control the classroom. Therefore students feel free to come late or leave early ..." (Student 6)

\section{Dominant individual and organisational culture}

According to teachers' experiences, there are factors that affect the incidence and management of uncivil behaviour. These factors included organisational culture, students' and teachers' personal characteristics and beliefs, and university nature. Norm difference between teachers and students was another factor interfering with incivility management.

\section{Students' personal characteristics and beliefs}

Among factors affecting incivility, participants highlighted different contexts where they grew up, influence of public 
media, family's educational status and resistance to change in some cases. In other cases, non-educational activities affected their behaviours-for example, the management of personal life problems, preference of employment over studying and family life over educational tasks, involvement in economic problems and coping with emotional problems. Note a participant's comments below:

“... he used to sit at the back row of the classroom; then, he turned on his music player, with really lower voice, but played bad damn music ..." (Student 4)

\section{Teachers' personal characteristics and beliefs}

Teachers' beliefs, attitudes, values, interest, experiences and performance in the field of education played important roles in managing incivility. Commitment to professionalism and accountability had a crucial role in incivility management and prevention. Note a participant's comments below:

“... I do many things to control their behaviour, but I am not concerned about my students liking me or not; whether they will like me or not, this is unimportant because whatever I do is based on my own belief." (Teacher 1)

\section{University nature}

University nature including dominant atmosphere, institutional and management policies and teaching styles of teachers affected the management of incivility. Also, the teachers' assessment approaches and their particular methods for a certain course were influential in effective management of incivility. Note a participant's comments below:

“... but here, I directly reminded the student of her inappropriate behaviour on the basis of the university rules and regulations; I mean, quite outspoken, I reprimanded her about the dress code and bad behaviour." (Teacher 7)

\section{Variation of norms for students and teachers}

Diversity in norms and values among students and teachers and the gap between generations led to uncivil behaviour. Some behaviours such as bullying others and dominating the classroom were valuable and enjoyable to students, although teachers considered them uncivil. Note a participant's comments below:

"... for students, a favourite student is the one who keeps good relations with instructors, studies less but gets higher marks, sometimes stands on the front and shows off against teachers and students, a student who is spontaneous and quick-witted ... these are not the values we instructors wish to develop in students ..." (Teacher 12)

\section{Guided democracy}

The basic psychosocial process in managing incivility was guided democracy which included six subcategories (table 2). Teachers were concerned about incivility due to their sense of responsibility, and they tended to give feedback to students' misconducts in one way or another. On the incidence of incivility, teachers resorted to various strategies based on the severity of the case, the time and place of its occurrence, keeping a respectful treatment of the class and the person, using decisive measures, passive acceptance or disregarding minor misbehaviors, private advice, delayed advice or other strategies to correct their behaviour. Although students usually enjoyed a democratic and less stressful environment, they had to comply with regulations. If students violated the rules accidentally or unintentionally, teachers usually provided them with advice to correct their misconduct in both strict and lenient manners, occasionally with a little humour and lessened strictness.

\section{Teachers' accountability}

Establishing discipline and order in classrooms and clinical training centres with an emphasis on course goals and prioritising scientific discussions indicates teachers' responsibility for course content and respective organisational requirements. In addition, teachers' supervision over students' theoretical and practical skills, their mutual behaviours in educational settings, their clinical interactions and sensitivity to students' incivility are all signs of a teacher's accountability. Note a participant's comments below:

“... I couldn't be mindless of whatever students wished to do, whenever they wish to come or leave, or avoid their care-taking duties in clinical training ... "

(Teacher 6)

\section{Cordiality strategies}

Some of the dimensions of cordiality strategies were stated as breaking the ice, respecting students' dignity and taking time for students' tutoring. In order to establish a comfortable atmosphere in dealing with students, statements were made about developing a sense of humour, letting students talk about their problems, helping them to calm down if serious things are already noted and even presenting humour. Teachers' routine practices for recognising students' dignity were stated as encouraging students, beginning classes on time, wishing to know students' feedback, asking for their opinions and treating them respectfully. Also, measures were reported to have been taken for students including encouraging students to get advice from teachers, trying to know them by their names, introducing the course and field training, counselling sessions, spending time with them and providing a sense of spiritedness. Note a participant's comments below:

“... I don't think I have to be hostile to their behaviours, but to return their greetings, not 
ignoring them if they are not accustomed to greeting or starting communication with me, I need to be independent from their reactions, and plan to make friends ..." (Teacher 9)

\section{Teaching-learning strategies}

Creating a satisfactory learning environment, adding to the attractions of classes and field trainings, mutual trust, extending professors' expectations, assessing academic discipline, being considerate towards education in general are aspects reported to lead to a favourable learning context. Note a participant's comments below:

"... I mean, when the content we present in classroom is good, and even not so excellent, students will be attracted ... they start to learn ..." (Teacher 2)

\section{Exigency decision-making}

Among their statements, teachers made references to some strategies. These strategies include avoiding immediate reaction to incivility, delayed response, providing continuous but less noticeable punishment to develop awareness to incivility in the long run, avoiding interference with the overall education of the intended student and making logical and constructive decisions. A sort of fake acceptance of uncivil students was expressed as the main cause of teachers' intentional ignorance of incivility. Other corrective strategies were stated as emphasis on a serious educational atmosphere, indirect treatments, general notes, private counselling, statement of rules and regulations from time to time and being strict on changing their behaviours. Note a participant's comments below:

“... while I was unhappy and irritated by that student's behaviour, I used to send him to the head of department so that they asked him for attending counselling sessions or signing a commitment paper, I didn't really want the student to fail the course, but wanted to create obstacles on their way so that a kind of awakening happens ..." (Teacher 2)

\section{Establishment of a collaborative atmosphere in education}

Seeking help from other colleagues in classroom management, modelling some successful colleagues, sharing experiences, discussing the issues and providing advice for each other were considered as steps in gaining more expertise for managing incivility. Conversely, sharing experiences between senior and junior students and modelling uncivil classmates sometimes contributed to the development of students' incivility. Note a participant's comments below:

“... at that time, I really didn't know what to do because I was strange at that particular setting, a parttime instructor, immediately I went to the head of the department and asked what really they did in such cases, and how they managed those disruptions ..." (Teacher 11)

\section{Intelligent self-regulation}

Intelligent self-regulation may manifest in experience-based practices, moderating one's managing behaviours and self-consciousness. Attitudinal variations towards disruptive behaviour in the long run, enhanced tactfulness, knowing behavioural models, converting threats to opportunities and studying classroom management booklets were among the factors stated to be contributing to incivility management. Teachers' revision of their strict disciplinary rules, balancing opportunities and educational activities, adopting the best possible teaching methods and strategies, applying modern ways in upcoming occasions, practicing for better behaviours and situational tailoring of the organisational regulations all show one's capability in managing incivility. Part of teachers' management capability was manifested in their self-consciousness in localising one's capabilities, developing awareness to one's behaviour and imagining its outcomes, and preventing its emergence through deeply thought-out solutions. Note a participant's comments below:

“... as our experiences increased, we were skillful in teaching the content; we started getting more familiar with how to treat students; we came to an understanding that we needed to value students, to keep good relations with them, to listen to their stories, and to value them ..." (Teacher 17)

\section{Movement to professionalism}

This category signifies the outcome of guided democracy, according to which students and teachers are expected to reach an adequate level of professionalism in their profession. It contains four subcategories: students' educational engagement; students' thoughtfulness and reconsideration; promotion of teacher-student interaction; and the result of a win-win opportunity for both parties.

\section{Students' educational engagement}

Students' educational engagement was one of the outcomes of managing incivility, according to guided democracy. Teachers' appropriate incivility management guided students towards proper planning for their studies; they also had the opportunity to plan for research and extracurricular activities. Therefore, planning for educational improvement and empowerment provided them with further energy and zeal to avoid incivility involvements. Note a participant's comments below:

"... we had become more interested, and more energetically we attended our clinical training; we didn't waste the time mischievously ..." (Student 4)

\section{Students' thoughtfulness and reconsideration}

Students contemplated on their misbehaviour after receiving a teacher's advice, and usually found a solution for the problematic situation. Reconsideration of the behaviour made them eventually modify or correct their 
behaviour. This led them to change their attitude towards that specific behaviour, while hampering any bias, misunderstanding and hostility. One of the participants asserted that:

“... later you think about it, your anger has been $90 \%$ because of your own problems and only $10 \%$ is attributable to the instructor's words ..." (Student 7)

\section{Promotion of teacher-student interaction}

Appropriate management of behaviour can result in a reasonable interaction, where misbehaving students find themselves close to their teachers; accordingly, mistrust turns into mutual trust. Most students state that mutual understanding and sympathy follows rapport and empathy, and they tend to stop disruptive behaviours in order to gain the approval of their teachers. Note a participant's comments below:

“... now I feel those treatments we had with our students; they gradually turned to be in line with us and to respect us, and we were really satisfied with their behaviours ..." (Teacher 1)

\section{The win-win consequence for both parties}

Another outcome of appropriate conflict resolution is the win-win situation for both students and teachers, which was easily perceived as a cause for minimised worries and maximised sense of convenience. Learning how to control oneself, respecting others, and understanding the teacher when managing incivility were manifestations of a win-win situation. For teachers, such a consequence appeared to be realised in achieving the educational objectives as well as the emergence of a multidimensional development of students in all aspects including science, practice, ethics, etc. A sense of turning challenges into opportunities provided the teacher with tranquillity and solace. Therefore, success in teaching and learning is one of the most important outcomes of incivility management for teachers and students.

Note a participant's comments below:

“... in the next 15 sessions they really understood that they had to attend the classes, all 16 sessions; they were present; the first session caused this, even I had no roll call in some sessions; they were present ... ; they attended the class and I had the chance to deliver the content to them ..." (Teacher 12)

\section{DISCUSSION}

The present study was conducted to explore the management process used by teachers on university students' incivility. The findings led to the formation of guided democracy as the main strategy for incivility management. The deterioration of learning and dominant individual and organisational culture appeared to be the main categories which contributed to the psychosocial processes underlying the incivility. Finally, incivility management led to movement to professionalism.

Clark developed three themes out of a phenomenological study of incivility, including (1) faculty behaving in demeaning and belittling ways, (2) treating students unfairly and subjectively, and (3) forcing students to conform to unreasonable faculty demands. These findings seem to be in line with those of the present study regarding deterioration of learning due to defective academic performance. Deterioration of learning proved interfering in teachers' practices, as suggested in Clark's model. Clark also found three emotional themes in managing incivility including being shocked, feeling embarrassed and weak, and feeling discouraged and excited. ${ }^{23}$ The present study identified similar results where an outcome of inappropriate incivility management was a mutual loss for students and teachers. Another finding in the present study indicated that immature students manifested more incivility and hostility; similarly, Reio and Ghosh found younger students had more uncivil behaviours. ${ }^{24}$

A background factor in guided democracy for managing incivility was dominant individual and organisational culture. The dominant organisational environment in nursing faculties tended to affect the incidence of incivility to a remarkable extent. Manners of enacting rules, the culture was dominating the individuals, and personal characteristics of students and teachers were also influencing incivility management. In line with these findings, Ibrahim and Qalawa explored factors affecting nursing students' incivility, including the faculty environment, political sphere and teachers themselves. $^{25}$

In guided democracy, management of incivility is guided by the type and severity of the behaviour in a democratic setting; however, teachers did not overlook behaviours and tended to supervise students' behaviour and help them to establish a relaxing and calm environment for teaching and learning. Similarly, Shanta and Eliason applied a model of empowerment in managing incivility by employing communication, equal rights and authority, independence and responsiveness. The concepts in Shanta and Eliason's model were almost similar to those of our guided democracy, since students enjoyed degrees of freedom in the latter too (almost equivalent to independence). The concept of communication in the former may also correspond to strategies like friendship, time specification and respecting learners. The decisiveness aspect in guided democracy indicates that learners are expected to be responsive to the rules and regulations of the teaching-learning environment; otherwise, teachers will use their authority to control the chaotic situation. The only difference between guided democracy and Shanta and Eliason's model of empowerment mayy be found in the equal rights and authority for both parties. However, in our study, justice and impartiality in treating every individual learner may correspond roughly to that concept. ${ }^{26}$ 
Another model addressing incivility and disruptive behaviour among healthcare providers was proposed by Holloway and Kusy in a system-oriented approach based on organisational, team and individual strategies. Almost equivalent to their organisational strategies, guided democracy contained a subcategory of dominant individual and organisational culture where managing students' incivility was likely to be realised under the circumstances governing the organisation and individual. Almost similarly, intelligent self-regulation was considered as a facilitative condition in guided democracy, where the interaction between team members and the counselling nature of this subcategory can drastically contribute to the proper management of incivility. Moreover, an approximate correspondence may be found in individual strategies ${ }^{27}$ and guided democracy, where accountability of the teachers is highlighted in managing incivility.

Teaching-learning strategies were also among the dimensions of guided democracy. Success in incivility management was mostly achieved by teachers, expert deliverers of the course content and those who successfully involved students in the learning process. Therefore, participants in the present study referred to this aspect as teaching-learning strategy and respecting learners. Similarly, by referring to active learning methods which really facilitate the learning experience, Clark contended that such strategies enhanced the faculty-student interaction leading to the development of better communications, social skills and educational efficiency, where the occurrence of incivility appears to be less likely. ${ }^{21}$

Another aspect of guided democracy indicated by the findings was reflection, through which the consideration of students' disruptive behaviours led teachers to intelligent self-regulation; this appears to be in line with Clark and Davis Kenaley who presented a model of teacher empowerment for managing incivility. ${ }^{28}$ Similarly, Kuhlenschmidt and Layne contend that faculty-student interaction helps the teacher to know the worries and fears of students, to understand them and to explore factors leading to incivility. Moreover, discussing the issue with an experienced colleague can improve such skills. ${ }^{20}$ As a major dimension in thoughtful interaction, self-reflection was also suggested in the guided democracy. Hood et al pointed to the nursing students' interaction with peers from other disciplines and found it to be a strategy which helped them to move towards more mature behaviours and professionalism. ${ }^{29}$

Another aspect in guided democracy was important decision-making by which teachers allowed some liberty in the classroom atmosphere. Most inexperienced teachers adopt this strategy. Hernandez and Fister also considered this issue and pointed to the silence strategy in order to prevent incivility. Teachers usually avoid discussing these problems with other colleagues, neither about their feelings nor experiences. ${ }^{9}$ This could be attributed to their own sense of disqualification, lack of responsibility and shame. Moreover, in cases with an embarrassing experience, the organisational managers may be blamed for showing lenience in managing students' incivility. However, adopting the strategies of guided democracy, teachers can help students start reconsideration of their behaviours, understand the effect of most incivility cases in learning and teaching, even convince peers to admit their faults and to apologise, and initiate a more cordial relationship with the teachers. Promotion of professionalism and the win-win strategy may come true if the teacher-student interaction is truly guided towards true teaching and learning, with no chance and space for incivility.

\section{Limitations of the study}

Although enjoying purposive sampling and maximum diversity among knowledgeable and experienced participants, since this study only focused on discovering the experiences of Iranian nurse students and teachers, further studies in other cultures are needed to expand this body of knowledge and make the results more generalisable.

\section{CONCLUSION}

The use of guided democracy in managing incivility provides a fresh look at the contribution of nursing students' incivility to their unprofessional practice in educational settings. The findings inform us about the incivility structure and process, psychosocial issues associated with it, facilitating and debilitating factors associated with students and teachers' behaviours as well as strategies to minimise incivility. The results can be applied in faculty empowerment programmes (both in-service and pre-service). It has shed light on the incivility issue and its various aspects to some extent; however, it still requires further investigation, particularly with other disciplines and cultures.

Acknowledgements The present article is extracted from a $\mathrm{PhD}$ dissertation approved by MUMS Vice- Chancellor for Research (approval code 921903) and financially supported by MUMS and School of Nursing and Midwifery, Mashhad, Iran. The authors thank all the teachers and students who participated in the study, and also Carol E Fletcher for her help in improving the language and methodology of this article.

Contributors All authors designed the study. MR and El were responsible for data collection and data analysis under the supervision of HKM. MR and El prepared the manuscript draft. All authors participated in the edition of the manuscript based on editor and reviewers' comments. HKM edited and approved the final manuscript. All authors had full access to all of the data in the study.

Funding The present study is financially supported by Mashhad University of Medical Sciences.

Competing interests None declared.

Patient consent None decalred.

Ethics approval The Ethics Committee of Mashhad University of Medical Sciences (No. 921903, 2014/08/03)

Provenance and peer review Not commissioned; externally peer reviewed. Data sharing statement No additional data are available.

Open Access This is an Open Access article distributed in accordance with the Creative Commons Attribution Non Commercial (CC BY-NC 4.0) license, which permits others to distribute, remix, adapt, build upon this work non-commercially, and license their derivative works on different terms, provided the original work is 
properly cited and the use is non-commercial. See: http://creativecommons.org/ licenses/by-nc/4.0/

(C) Article author(s) (or their employer(s) unless otherwise stated in the text of the article) 2017. All rights reserved. No commercial use is permitted unless otherwise expressly granted.

\section{REFERENCES}

1. Clark CM. Faculty and student assessment of and experience with incivility in nursing education. J Nurs Educ 2008;47:458-65.

2. Rad M, Ildarabadi $\mathrm{EH}$, Moharreri $\mathrm{F}$, et al. A study of incivility in the Iranian nursing training system based on educators and students experiences: a quantitative content analysis. Glob J Health Sci 2014;7:203.

3. Robertson JE. Can't we all just get along? A primer on student incivility in nursing education. Nurs Educ Perspect 2012;33:21-6.

4. Swinney L, Elder B, Seaton Lloyd "Pat". Incivility in the Accounting Classroom. Am J Bus Educ 2005;3:1-16.

5. Axup T, Gersch I. Challenging behaviour: the impact of challenging student behaviour upon teachers' lives in a secondary school: teachers' perceptions. Br J Special Educ 2008;35:144-51.

6. McKinney SE, Campbell-Whately GD, Kea CD. Managing student behavior in urban classrooms: the role of teacher ABC assessments. The Clearing House: A Journal of Educational Strategies, Issues and Ideas 2005;79:16-20.

7. Luparell $S$. The effects of student incivility on nursing faculty. J Nurs Educ 2007;46.

8. Karimi Moonaghi H, Rad M, Torkmannejad Sabzevari M. Management of challenging behavior (incivility) among medical students. Future Med Educ J 2014:4:41.

9. Hernandez TJ, Fister DL. Dealing with disruptive and emotional college students: a systems model. J Coll Couns 2001;4:49-62.

10. Heydari A, Rad M, Rad M. Evaluating the incivility between staff nurses and matrons employed in Iran [Procena nepristojnog pona?anja u me? usobnom odnosu medicinskih sestara i glavnih medicinskih sestara zaposlenih u Iranu]. Acta Facultatis Medicae Naissensis 2015;32:137-46.

11. Feldmann LJ. Classroom civility is nnother of our instructor responsibilities. College Teaching 2001;49:137-40.

12. Schaeffer A. The effects of incivility on nursing education, 2013.

13. Luparell S. The effects of student incivility on nursing faculty. J Nurs Educ 2007;46:15-19.
14. Hossein KM, Fatemeh D, Fatemeh OS, et al. Teaching style in clinical nursing education: a qualitative study of Iranian nursing teachers' experiences. Nurse Educ Pract 2010;10:8-12.

15. Borhani FME, Abbaszadeh A. Nurses qualified professional ethics, needs and challenges in ethical education. Iranian J Med Ethics History Med 2009;2:27-38.

16. Estes B, Workplace incivility: impacts on individual and organizational performance. Human Resource Develop Rev 2008;7;2: 218-240

17. Del Prato D. Students' voices: the lived experience of faculty incivility as a barrier to professional formation in associate degree nursing education. Nurse Educ Today 2013;33:286-90.

18. Farrell GA, Bobrowski C, Bobrowski P. Scoping workplace aggression in nursing: findings from an Australian study. J Adv Nurs 2006;55:778-87.

19. Seidman A. The learning killer: disruptive student behavior in the classroom. Reading Improvement 2005;42:40.

20. Kuhlenschmidt SL, Layne LE. Strategies for dealing with difficult behavior. New Directions for Teaching and Learning 1999;1999:45-57.

21. Clark CM. Faculty field guide for promoting student civility in the classroom. Nurse Educ 2009;34:194-7.

22. Strauss A, Corbin J. Basics of qualitative research: techniques and procedures for developing grounded theory. Sage Publications, 1998.

23. Clark C. Student perspectives on faculty incivility in nursing education: an application of the concept of rankism. Nurs Outlook 2008;56:4-8.

24. Reio TG, Ghosh R. Antecedents and outcomes of workplace incivility: implications for human resource development research and practice. Human Resource Develop Q 2009;20:237-64.

25. Ibrahim SA, Qalawa SA. Factors affecting nursing students' incivility: as perceived by students and faculty staff. Nurse Educ Today 2016;36:118-23.

26. Shanta LL, Eliason AR. Application of an empowerment model to improve civility in nursing education. Nurse Educ Pract 2014;14:82-6.

27. Holloway E, Kusy M. Systems approach to address incivility and disruptive behaviors in health-care organizations. Adv Health Care Manag 2011;10:239-65.

28. Clark CM, Davis Kenaley BL. Faculty empowerment of students to foster civility in nursing education: a merging of two conceptual models. Nurs Outlook 2011;59:158-65.

29. Hood K, Cant R, Baulch J, et al. Prior experience of interprofessional learning enhances undergraduate nursing and healthcare students' professional identity and attitudes to teamwork. Nurse Educ Pract 2014;14:117-22. 